\title{
Electrodynamic model of relict radiation scattering by vegetation cover to improve the accuracy of soil moisture measurement by microwave radiometer
}

\author{
Sergey Chigikov ${ }^{1}$, Dmitri Gorbachev ${ }^{1,2}$, Alexey Taradin ${ }^{3, *}$, and Maria Sidorova ${ }^{3}$ \\ ${ }^{1}$ Bauman Moscow State Technical University, 105005, 2-ya Baumanskaya st., 5, Moscow, Russia \\ ${ }^{2}$ Open Company «Miraglobex», 115201, Kashirskiy pass, 13 room 12, Moscow, Russia \\ ${ }^{3}$ Moscow Institute of Physics and Technology, 141701, Institutskiy per., 9, Dolgoprudny, Russia
}

\begin{abstract}
The possibilities of electrodynamic model of relict radiation scattering by vegetation cover to improve the accuracy of soil characteristics measurement by microwave radiometer are demonstrated. The dependence of the reflective characteristics of the soil-vegetation system on the type and parameters of vegetation cover is considered. The accuracy of measuring the radio brightness temperature of the soil is estimated depending on the type and characteristics of the vegetation cover.
\end{abstract}

\section{Introduction}

Then measuring the soil moisture and surface temperature using a microwave radiometer [1], vegetation cover significantly influence on the measurement results [2], as vegetation partially reflects and partially absorbs the relict radiation of the celestial sphere, as well as shields its own radiothermal radiation of the soil. Modeling of reflective and absorbing characteristics of vegetation in active and passive remote sensing of the earth in order to determine the soil moisture and vegetation parameters has described by many researchers [3-5]. Taking into account the influence of vegetation cover to clarify the results of measuring soil moisture and temperature is an urgent task and requires the development of new methods for modeling the interaction of an electromagnetic wave with the surface of the earth covered with vegetation L-band microwave radiometer, designed for remote measurement of soil moisture in a layer up to one meter depth from the board of the unmanned helicopter.

Microwave radiometer antenna, located on unmanned helicopter, directs to the earth surface so that the antenna paten axis have some angle with vertical line. In this case the microwave radiometer output signal will be the sum of two waves, one is one earth thermal emission and second the sky relict emission reflected from earth surface. The location of microwave radiometer is shown on Fig.1.

\footnotetext{
*Corresponding author: aleksey.taradin@phystech.edu
} 


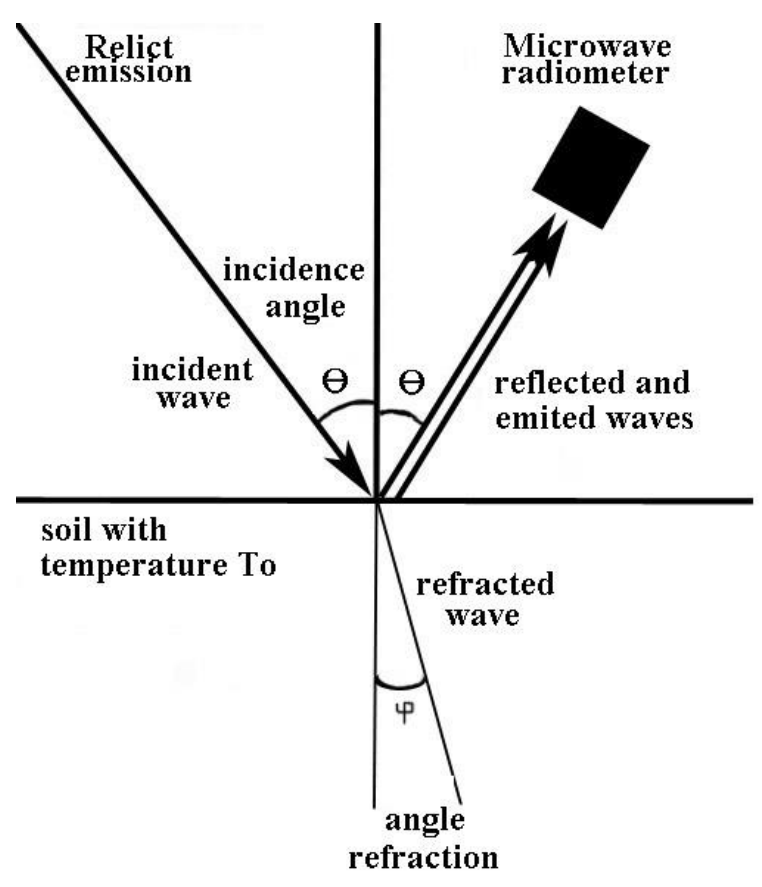

Fig. 1. The location of microwave radiometer.

The microwave radiometer must be able to measure the brightness temperatures both vertical and horizontal polarized waves. The radio brightness temperatures $T_{V}$ and $T_{H}$ are measured by radiometer on vertical and horizontal polarizations. From the measured values and regarding the known soil temperature $\mathrm{T}_{0}$ and the sky relict radiation temperature $\mathrm{T}_{\mathrm{S}}$, it is possible to calculate the reflection coefficients for the vertically polarized wave $r_{V}$ and horizontally polarized wave $\mathrm{r}_{\mathrm{H}}(1)$

$$
\begin{aligned}
& T_{V}=T_{0} \cdot\left(1-r_{V}\right)+T_{S} \cdot r_{V} \\
& T_{H}=T_{H} \cdot\left(1-r_{H}\right)+T_{H} \cdot r_{H} \\
& r_{V}=\frac{T_{0}-T_{V}}{T_{0}-T_{S}} ; r_{H}=\frac{T_{0}-T_{H}}{T_{0}-T_{S}}
\end{aligned}
$$

Reflection coefficients $r_{V}$ and $r_{H}$ are also determined by Fresnel formulas (2)

$$
r_{V}=\left|\frac{\varepsilon \cdot \cos (\Theta)-\sqrt{\varepsilon-\sin ^{2}(\Theta)}}{\varepsilon \cdot \cos (\Theta)+\sqrt{\varepsilon-\sin ^{2}(\Theta)}}\right|^{2} \quad r_{H}=\left|\frac{\cos (\Theta)-\sqrt{\varepsilon-\sin ^{2}(\Theta)}}{\cos (\Theta)+\sqrt{\varepsilon-\sin ^{2}(\Theta)}}\right|^{2}
$$

Formulas (2), at a known incidence angle $\Theta$ and calculated from the measured values $r_{V}$ and $r_{H}$, represents equations where the unknown is the permittivity of the soil, which in general is a complex value $\varepsilon=\varepsilon_{1}+\mathrm{i} \varepsilon_{2}$. The joint solution of the system of equations (2) makes it possible to determine the real and imaginary parts of the soil permittivity, which depends on the soil moisture value.

When the soil surface covered by vegetation, the brightness temperatures reflected and emitted waves are not exactly defines by formulas (1) and (2). So the accuracy of soil moisture calculation will be reduced. To improve the accuracy it is necessary to regard the scattering relict emission on vegetation and the absorption soil emitted wave in vegetation. 
This is difficult problem not completely solved up to current moment. One of passible approach to solve the vegetation influence problem is to simulate the electro-magnetic wave scattering on dielectric structures in mathematical model. To show the results of such modeling in simplest case is the purpose of this article.

\section{Electrodynamic model of relict radiation scattering}

To simulate the wave scattering on the grass model has been designed (Fig.2).
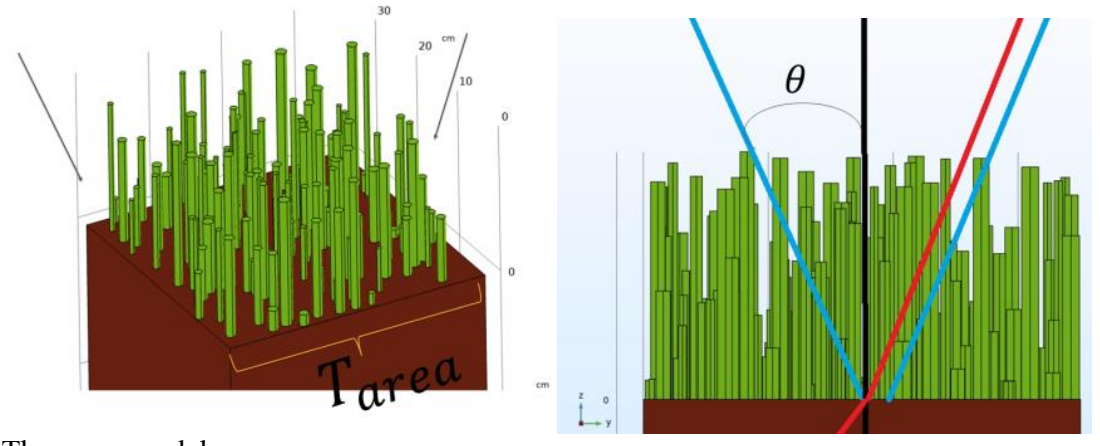

Fig. 2. The grass model.

The grass represents like cylinders of dielectric material with different lengths and diameters. The probability of parameters distribution was considered as constant. The number of grass blades per unit area are constant. A plane electromagnetic wave on vertical or horizontal polarization in the L-band falling on a flat surface at some angle to the vertical is simulated.. The parameters of the electromagnetic wave reflected from a flat surface with cylinders and their dependence on the input parameters of the model are calculated. A plane electromagnetic wave of vertical or horizontal polarization propagating from a flat surface into open space through a system of cylinders simulating the soil's own radiothermal radiation is also simulated. The parameters of the electromagnetic wave passed through the "grass" layer are calculated.

\section{Simulation results}

The field distribution was calculated in «Comsol Multiphysics». The results of calculations for incidence angels 45 and 0 degrees are represented on Fig. 3.

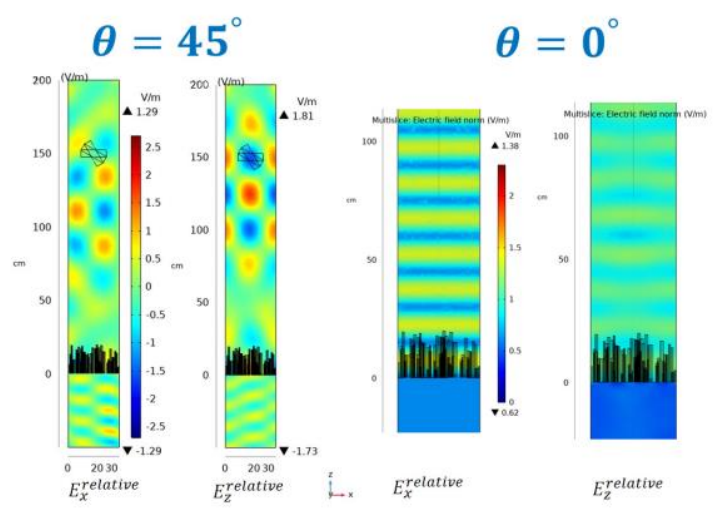

Fig. 3. The field distribution for incidence angels 45 and 0 degrees. 
The scattering cross-section chart is representing on Fig.4.

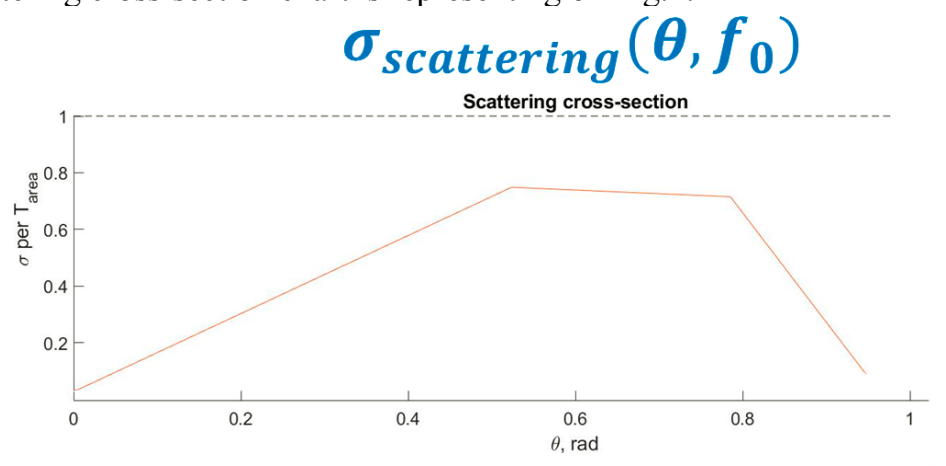

Fig. 4. Scattering cross-section chart.

Similar calculations were made for different incidence angles, different wave polarizations, different values of grass simulation parameters. It is impossible to present all the results in a short paper. But the general analysis of the results showed that even in the L-band in some cases the scattering cross-section has a significant value. It is not possible to measure soil moisture, as the soil is screened by vegetation, and influence is different for both polarizations. In any case, the presence of vegetation makes significant errors when the brightness temperatures is measuring. In the presence of vegetation, a priori information about the nature of vegetation and appropriate models are required to account for its influence.

\section{Conclusion}

As a result of the research it is possible to make the following conclusions:

- the presence of vegetation on the soil surface impairs the accuracy of radiometric meters of soil temperature and humidity;

- additional a priori information on vegetation parameters is required to account for the shielding effect of vegetation cover;

Research was supported by a grant of the Foundation for the Advancement of Small Businesses in Science and Technology (Innovation Promotion Fund) (Contract No. 2886GS1/45415).

\section{References}

1. I.A. Sidorov, V.A. Plushev, A.P. Soldatenko, A.D. Reschikov, D.A. Gorbachev, About the possibility of using a group of UAVs to make maps of soil moisture in precision farming, CriMiCo 2018 - 28th International Crimean Conference Microwave and Telecommunication Technology, Sevastopol, , Volume 7, pp. 1610-1616 (5-15 September, 2018)

2. I.A. Sidorov. and others edited By V.S. Verba, Yu.V. Gulyaev, A.M. Shutko, V.F. Krapivin. Microwave radiometry of land and water surfaces: from theory to practice. Sofia, Academic publishing house named after Professor Marin Drinov, 295 p. (2014)

3. V.N. Sagalovich, E.Ya. Falkov, T.I. Tsareva, Determination of soil moisture using active microwave remote sensing data in the L-band. Earth exploration from space. No. 1., pp. 3-6. ISSN: 0205-9614 (2007)

4. G.P. Kulemin, S.V. Yatsevich, Interrelation of backscattering of microwave radio waves with parameters of vegetation cover and open soils at remote sensing methods, Achievements of Modern Radioelectronics, №3 pp. 24-34. ISSN: 2070-0784 (2004)

5. T.J. Jackson, D.Chen, M.Cosh et al. Vegetation water content mapping using Landsatdata derived normalized difference water index for corn and soybeens, Remote Sensing of Invironment, V. 92, pp.475-482. . ISSN: 0034-4257 (2004) 